\title{
ON UNKNOTTING NUMBERS AND KNOT TRIVADJACENCY
}

\author{
A. STOIMENOW
}

\begin{abstract}
We prove for rational knots a conjecture of Adams et al. that an alternating unknotting number one knot has an alternating unknotting number one diagram. We use this then to show a refined signed version of the Kanenobu-Murakami theorem on unknotting number one rational knots. Together with a similar refinement of the linking form condition of Montesinos-Lickorish and the HOMFLY polynomial, we prove a condition for a knot to be 2-trivadjacent, improving the previously known condition on the degree-2-Vassiliev invariant.

We finally show several partial cases of the conjecture that the knots with everywhere 1-trivial knot diagrams are exactly the trivial, trefoil and figure eight knots. (A knot diagram is called everywhere $n$-trivial, if it turns into an unknot diagram by switching any set of $n$ of its crossings.)
\end{abstract}

\section{Introduction}

The concept of $n$-trivadjacency (see [5], [20]) has been recently introduced as a specialization of Gusarov's notion of $n$-triviality [17] (introduced in the context of Vassiliev invariants [6]), and generalization of the property unknotting number one [27], [39], [48] (with which it coincides for $n=1$ ), and is also related to other unknotting operations [3], [4], [35]. In [5], N. Askitas and E. Kalfagianni studied the case $n \geq 3$, obtaining several results on $n$-trivadjacent knots in these cases (in particular, all such knots have unit Alexander polynomial and are 5-trivial). These conditions, on the other hand, strongly restrict the class of such knots (the simplest non-trivial examples have very likely not less than 23 crossings and certainly not less than 14 crossings). The case $n=2$ is rather different, since the results of [5] do not apply, and in fact there are simple examples of 2-trivadjacent knots (the trefoil and figure-8 are such). Therefore, beside the classic unknotting number one case $n=1$, the 2-trivadjacent case is most interesting to study.

In this paper we will deal with the cases $n \leq 2$. We start in $\S 3$ with $n=1$ and prove in $\$ 3.2$ a refined (signed) version of the unknotting number theorem of Kanenobu-Murakami [22] for rational knots. This theorem uses another result, proved previously in $\$ 3.1$, confirming for rational unknotting number

Received March 4, 2002; in revised form December 15, 2002. 
one knots a conjecture due to Bernhard, Jablan and Adams, concerning the existence of an unknotting number one minimal (crossing number) diagram.

Then in $\S 4$ we consider $n=2$ and prove a condition for 2-trivadjacency using the skein (HOMFLY) polynomial [18], which generalizes the previously known condition $\left|v_{2}\right| \leq 1$ in the $v_{2}= \pm 1$ case ( $v_{2}$ denotes the degree-2Vassiliev invariant). Together with the signed refinement of the theorem of Lickorish [27] in [43], this allows to decide about 2-trivadjacency for most knots in Rolfsen's tables [38, appendix], with 19 exceptions.

Finally, in $\$ 5$ we consider a generalization of $n$-triviality we call everywhere- $n$-triviality, and consider in particular the case $n=1$ (where the condition on a knot diagram means that it remains/becomes unknotted after switch of any arbitrary crossing). We present some computational evidence for a conjecture stating that the only non-trivial knots possessing everywhere 1-trivial diagrams are the trefoil and figure eight knot (and giving all these diagrams). We then apply the results of $\S 3$ to deduce a partial statement towards this conjecture.

\section{Preliminaries}

A knot diagram $D$ is an immersed oriented closed curve in the plane with transverse self-intersections, called crossings, each one of which is weighted by a sign indicating over- and underpassing strand. The set of all knot diagrams we denote by $\mathscr{D}$, so that $\mathscr{D} /\{$ Reidemeister moves $\}=\mathscr{K}$ is the set of all knots.

For a knot diagram $D$, and a subset $S$ of the set $C(D)$ of its crossings, let $D_{S}$ be the diagram obtained from $D$ by switching all crossings in $S$. Let $D_{p}=D_{\{p\}}$. The knot represented by a diagram $D$ we denote by $[D]$. The unknot we will write as $\bigcirc$.

The concept of $n$-triviality was introduced by Gusarov [17], who proved its fundamental importance in the study of Vassiliev invariants. (Here and in the sequel ' $\subset$ ' denotes a not necessarily proper inclusion.)

Definition 2.1. A knot diagram $D$ is called $n$-trivial, if there are subsets $S_{1}, \ldots, S_{n} \subset C(D)$ with $S_{i} \cap S_{j}=\varnothing$ for $i \neq j$, such that for any $\varnothing \neq$ $I \subset\{1, \ldots, n\}$ the diagram $D_{\cup_{i \in I} S_{i}}$ is an unknot diagram. A knot $K$ is called $n$-trivial, is it has an $n$-trivial diagram.

The concept of $n$-trivadjacency arises from $n$-triviality when demanding $\left|S_{i}\right|=1$.

Definition 2.2. A knot diagram $D$ is called $n$-trivadjacent, or $n$-adjacent to the unknot, if there is a subset $S \subset C(D)$ with $|S|=n$, such that $D_{S^{\prime}}$ is an unknot diagram for any $\varnothing \neq S^{\prime} \subset S$. A knot is called $n$-trivadjacent if it has an $n$-trivadjacent diagram. 
A related, but much more classical, concept is that of the unknotting number of diagrams and knots.

Definition 2.3. The unknotting number $u(D)$ of a diagram $D$ is defined as

$$
u(D):=\min \left\{|S|: S \subset C(D),\left[D_{S}\right]=\bigcirc\right\} .
$$

The unknotting number $u(K)$ of a knot $K$ is defined as

$$
u(K):=\min \{u(D):[D]=K\} .
$$

Note that a diagram of a non-trivial knot (i.e., not the unknot) has unknotting number 1 iff it is 1-trivadjacent. We will later apply tools developed to study unknotting number one to 2-trivadjacency.

Similarly, we have the crossing number of diagrams and knots.

Definition 2.4. The crossing number $c(D)$ of a diagram $D$ is defined as $|C(D)|$. The crossing number $c(K)$ of a knot $K$ is defined as

$$
c(K):=\min \{c(D):[D]=K\} .
$$

A diagram $D$ with $[D]=K$ and $c(D)=c(K)$ is called minimal (crossing number) diagram for $K$.

Definition 2.5. We call a diagram prime if there is no closed curve intersecting it transversely in exactly two points such that both its interior and exterior contain crossings of the diagram. (This closed curve is not to intersect the diagram at the crossings.) Otherwise the diagram is called composite. We call a knot prime if in any of its composite diagrams, one of the arcs separated by the closed curve is unknotted.

We will consider knots with special types of diagrams, studied for a long time.

Definition 2.6. A knot diagram is called alternating, if the strand passes all crossings alternatingly as under- and over-crossings. A knot is called alternating, if it has an alternating diagram.

A subclass of the alternating knots are the rational knots, which we define according to Conway [13].

Definition 2.7. A tangle diagram is a diagram consisting of strands crossing each other, and having 4 ends. A rational tangle diagram is the one that can be obtained from the primitive Conway tangle diagrams by iterated leftassociative product in the way displayed in figure 1. (A simple but typical 


\section{)}
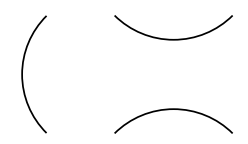

0

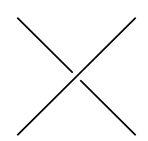

1

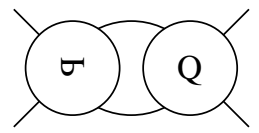

product $P Q$

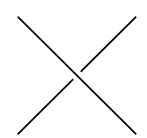

$-1$

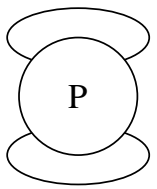

closure $\bar{P}$

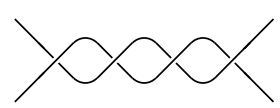

4

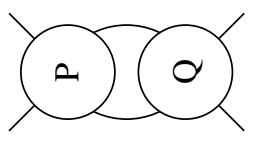

sum $P, Q$$$
\text { product } P Q
$$

FIGURE 1. Conway's tangles and operations with them. (The designation 'product' is very unlucky, as this operation is neither commutative, nor associative, nor is it distributive with 'sum'. Also, 'sum' is associative, but not commutative.)

example of a rational tangle is shown also there.) A rational knot is a knot having a diagram which is the closure of a rational tangle diagram. We call this diagram a rational knot diagram.

The left-associative product representation determining the rational tangle diagram is called the Conway notation for the rational knot.

The skein, or HOMFLY, polynomial $P$ is a Laurent polynomial in two variables $l$ and $m$ of oriented knots and links and can be defined by being 1 on the unknot and the (skein) relation

$$
\left.l^{-1} P(X)+l P(X)=-m P() \circlearrowright\right)
$$

Here the 3 diagram fragments are understood to represent the differing parts of otherwise identical link diagrams. We call the left resp. middle one a positive resp. negative crossing. The procedure by replacing any of those by the right diagram is called smoothing out.

The Jones polynomial $V$, and (one variable) Alexander polynomial $\Delta$ are obtained from $P$ by the substitutions

$$
\begin{aligned}
& V(t)=P\left(-i t, i\left(t^{-1 / 2}-t^{1 / 2}\right)\right), \\
& \Delta(t)=P\left(-i, i\left(t^{1 / 2}-t^{-1 / 2}\right)\right),
\end{aligned}
$$

hence these polynomials also satisfy corresponding skein relations.

For an account on the skein polynomial we refer to the papers [18] and [28]. (Note: our convention here for $P$ differs from [28] by interchange of $l$ and $l^{-1}$, that is, our $P(l, m)$ is Lickorish and Millett's $P\left(l^{-1}, m\right)$.) 
We will denote by $[P]_{m^{k}}$ the coefficient of $m^{k}$ in $P$ (this is a polynomial in l).

We continue by some discussion of nugatory crossings.

Definition 2.8. A crossing $p$ in a knot diagram $D$ is called nugatory, if $D_{p}$ and $D$ represent the same knot: $\left[D_{p}\right]=[D]$.

(This definition deviates from the commonly used one requiring there to be a closed curve in the plane intersecting $D$ transversely only in $p$.)

By $\operatorname{det}(K)$ we denote the determinant of $K$, given by $\left|\Delta_{K}(-1)\right|, \Delta_{K}$ being the Alexander polynomial of $K$.

Let $Z_{p}^{*}$ be the group of units of $Z_{p}=\mathbf{Z} / p \mathbf{Z}$. There is an equivalence (explained in [41]), related to some identities from the theory of elliptic forms, between the existence of representations of a natural number $p$ as the sum of the squares of two coprime numbers, the lack of (prime) divisors of $p$ of the form $4 k+3$, and the existence of square roots of -1 in the unit group $Z_{p}^{*}$ of the congruence ring.

\section{Rational knots of unknotting number one}

\subsection{The Adams-Bernhard-Jablan conjecture}

The fascination of unknotting numbers is the sharp contrast between the effort in defining them (as the minimal number of crossing changes necessary to perform in some diagram to create an unknot) and calculating them. Yet no tool is available to compute unknotting numbers, which is simultaneously satisfactory simple and generally applicable (see [25, table on p. 49], loc. cit. and related discussion).

Therefore, one was tempted to make conjectures about easy ways of determining unknotting numbers. For a while it was hoped that one could replace the infinite set on the right of (1) by a finite one, and compute the unknotting number of a knot correctly just from minimal (crossing number) diagrams, that is by

$$
\min \{u(D):[D]=K, c(D)=c(K)\} .
$$

However, this hope was disappointed by the example $10_{8}$ of Bleiler [9] and Nakanishi [36], for which the unknotting number is 2, yet (3) gives the value 3.

To salvage the situation, several subsequent conjectures expressed some weaker but hopefully more realistic desires, the most suggestive of which is to allow in between the crossing changes the freedom to change the knot diagram to a minimal one. 
Definition 3.1. For a knot $K$ let a modified unknotting sequence be a sequence $\left(D_{0}, \ldots, D_{n}\right)$ of length $n$, with (i) $\left[D_{0}\right]=K,\left[D_{n}\right]=\bigcirc$, (ii) $c\left(D_{i}\right)=c\left(\left[D_{i}\right]\right)$, and (iii) $\exists p_{i} \in C\left(D_{i}\right):\left[\left(D_{i}\right)_{p}\right]=\left[D_{i+1}\right]$ for $0 \leq i<n$. Define the modified unknotting number $u^{\prime}(K)$ as the smallest length $n$ of a modified unknotting sequence of $K$.

Then for the calculation of $u^{\prime}$ one still has to consider only finitely many diagrams, and one hopes for

Conjecture 3.2 (see [8], [21]). $u(K)=u^{\prime}(K)$ for any knot $K$.

A special case of this conjecture (for unknotting number one and alternating knots) was made by C. Adams.

Conjecture 3.3 (Adams, [1], question 4, p. 61). An alternating knot of unknotting number one has an alternating diagram of unknotting number one.

REMARK 3.4. By [23], [34], [46] for an alternating prime knot 'alternating diagram' is equivalent to 'minimal diagram'. If the alternating knot is not prime only the forward implication $\Rightarrow$ is true, but unknotting number one knots are prime [39], [50], so the conjecture of Adams is indeed a special case of the conjecture of Bernhard and Jablan.

REMARK 3.5. By the proof of the Tait flyping conjecture [33] any alternating diagram of some alternating knot has the same unknotting number, hence if an alternating knot has an alternating diagram of unknotting number one, the same is true for all its alternating diagrams.

REMARK 3.6. It has been pointed out by Jablan, that conjecture 3.3 (and hence conjecture 3.2) is in general false, if Nakanishi's table entry $u\left(9_{29}\right)=1$ is correct, see [25, table, p. 49]. So far, however, I have not seen an unknotting number one diagram of $9_{29}$.

Here we confirm the Adams conjecture (and so the Bernhard-Jablan conjecture) for rational knots.

THEOREM 3.7. Any rational knot of unknotting number one has an alternating diagram of unknotting number one.

Rational knots will be denoted according to Schubert [40] by $S(p, q)$ $(p>q>0,(p, q)=1)$ or according to Conway [13] by $C\left(a_{1}, \ldots, a_{r}\right)$. To explain the relation between both notations, define the iterated fraction (IF) 
of a sequence of integers $a=\left(a_{1}, \ldots, a_{r}\right)$ recursively by

$$
\begin{aligned}
I F\left(a_{1}\right) & :=a_{1}, \ldots, I F\left(a_{1}, \ldots, a_{r-1}, a_{r}\right) \\
& :=\frac{1}{I F\left(a_{1}, \ldots, a_{r-1}\right)}+a_{r}=a_{r}+\frac{1}{a_{r-1}+\frac{1}{\cdots+\frac{1}{a_{1}}}} .
\end{aligned}
$$

It will be helpful to extend the operations ' + ' and ' $1 /$.' to $\mathrm{Q} \cup\{\infty\}$ by $1 / 0=\infty$, $1 / \infty=0, k+\infty=\infty \forall k \in \mathrm{Z}$. In this sense, $I F$ is a map $(\forall r \in \mathbf{N})$

$$
I F: Z^{r} \longrightarrow \mathrm{Q} \cup\{\infty\} .
$$

Then $S(p, q)=C\left(a_{1}, \ldots, a_{r}\right)$ with $I F\left(a_{r}, \ldots, a_{1}\right)=p / q$. (By convention, $a_{1}, \ldots, a_{r}$ may sometimes be ordered backwards in other papers.)

The rational diagram associated to the Conway form $C\left(a_{1}, \ldots, a_{r}\right)$ is that of the product $\left(\ldots\left(\left(a_{r} a_{r-1}\right) a_{r-2}\right) \ldots\right) a_{1}$ in the tangle construction of figure 1 .

Example 3.8. The rational tangle $-2-342$ in figure 1 closes to a rational knot with Conway notation $C(2,4,-3,-2)$, whose associated iterated fraction is

$$
I F(-2,-3,4,2)=2+\frac{1}{4+\frac{1}{-3+\frac{1}{-2}} .}
$$

When considering orientation of the ambient space, $S(p, q)$ and $S\left(p^{\prime}, q^{\prime}\right)$ are equivalent iff $p=p^{\prime}$ and $q=q^{\prime}$ or $q q^{\prime} \equiv 1 \bmod p$. We denote this equivalence by $S(p, q) \doteq S\left(p^{\prime}, q^{\prime}\right)$, i.e. $S(p, q) \doteq S\left(p, q^{ \pm 1}\right)$, the inversion meant in $\mathbf{Z}_{p}^{*}$. If the orientation of the ambient space is not considered, then additionally $S(p, q)$ is equivalent to $S\left(p,-q^{ \pm 1}\right):=S\left(p,(p-q)^{ \pm 1}\right)$. We denote this weaker equivalence by ' $\sim$ '. These equivalences follow from Schubert's classification [40], which is explained also in textbooks like [11], [38].

Our proof of theorem 3.7 decisively uses some work done in this context by Kanenobu and Murakami [22].

THEOREM 3.9 (Kanenobu and Murakami [22]). A rational knot is of unknotting number one if and only if it can be expressed as $C\left(a, a_{1}, \ldots, a_{r}, \pm 2,-a_{r}\right.$, $\left.\ldots,-a_{1}\right)$ for some integers $a, a_{1}, \ldots, a_{r} \neq 0$.

More specifically we will use some details of their proof, which we will indicate below. 
Proof OF TheOREM 3.7. Consider in more detail the argument in [22, proof of theorem 1, (ii) $\Rightarrow$ (iii)]. Take a rational unknotting number one knot $K$ and fix the sign $\varepsilon \in\{ \pm 1\}$ appearing in the proof. The symmetry of the expression of $K$ in $m$ and $n$ allows us to take $m$ to be the bigger one and hence to be able to choose $t$ of either signs without spoiling $a \neq 0$.

First consider the case where $a$ is of the same sign as $\varepsilon$. Choose $t$ also to be of sign $\varepsilon$. Then, taking the expression of $n / t$ as iterated fraction with all numbers of the same sign, one obtains according to Kanenobu and Murakami an expression $K=C\left(a, a_{1}, \ldots, a_{r}, 2 \varepsilon,-a_{r}, \ldots,-a_{1}\right)$ with $a, a_{1}, \ldots, a_{r}$ all of sign $\varepsilon$. (An illustration of these knots is given in [22].) But now (unless we are in the uninteresting twist knot case $n=0) \varepsilon I F\left(-a_{1}, \ldots,-a_{r}\right)<$ -1 , hence, expressing $1<\varepsilon I F\left(-a_{1}, \ldots,-a_{r}, 2 \varepsilon\right)<2$ as iterated fraction with all numbers of sign $\varepsilon$, we have $C\left(\varepsilon, \varepsilon a_{1}^{\prime}, \ldots, \varepsilon a_{r}^{\prime}\right)$ with all $a_{i}^{\prime}>$ 0 . Now, switching one of the crossings in the (first) groups of the tangles $C\left(2 \varepsilon,-a_{r}, \ldots,-a_{1}\right)$ and $C\left(\varepsilon, \varepsilon a_{1}^{\prime}, \ldots, \varepsilon a_{r}^{\prime}\right)$ (those of 1 or 2 crossings resp.) gives the same tangle and, after adding the remaining twists and closing up, the same knot, which from former expression is seen to be the unknot. Hence the diagram $C\left(a, a_{1}, \ldots, a_{r}, \varepsilon, \varepsilon a_{1}^{\prime}, \ldots, \varepsilon a_{r}^{\prime}\right)$ is alternating and unknots by switching the middle $\varepsilon$ to $-\varepsilon$, and we are done.

Let $a$ and $\varepsilon$ be of different signs. Now, observe that so far in fact we showed that the tangle $C\left(a_{1}, \ldots, a_{r}, 2 \varepsilon,-a_{r}, \ldots,-a_{1}\right)$ has a representation $C=C\left(b_{1}, \ldots, b_{s}\right)$ with all the entries $b_{i}$ of the same sign (equal to this of $a_{1}$ ) and $s=2 r+1$, such that the tangle diagram reduces to a diagram of the 0 -tangle by changing a crossing $p$, and hence unknots the closed tangle diagram $C\left(a, b_{1}, \ldots, b_{s}\right)$. Adding the $a$ half twists to $C\left(b_{1}, \ldots, b_{s}\right)$ gives an alternating diagram if $a$ and $a_{1}$ have the same sign. This is the case we already discussed.

In the opposite case consider the bijection map of tangles

$$
C\left(b_{1}, \ldots, b_{s}\right) \longleftrightarrow C\left(1, b_{1}-1, b_{2}, \ldots, b_{s}\right)
$$

for $b_{1}>1$. Switching the image crossing of $p$ in the image tangle of $C$ again gives the 0 -tangle because of the identity

$$
1 / I F\left(b_{s}, \ldots, b_{1}\right)+1 / I F\left(b_{s}, \ldots, b_{2}, b_{1}-1,1\right)=1 .
$$

The exceptional cases, where the performability of this crossing change may be spoiled by taking the image under the bijection (4) are only those of $r=0$ and $r=1,\left|a_{1}\right|=1$, and they can be checked directly. The case $r=0$ gives a twist knot, and for $r=\left|a_{1}\right|=1$ we also obtain a twist knot, as $a_{1}$ and $\varepsilon$ are of the same sign, and $\left(a_{1}, 2 \varepsilon,-a_{1}\right)= \pm(1,2,-1)=( \pm 2)$. Otherwise, $p$ exists also in the tangle on the right of (4), and is not the crossing of the initial ' 1 '. 
But now because of the same identity (5) we have

$$
C\left(a,-\operatorname{sgn}(a), b_{2}, \ldots, b_{s}\right)=C\left(a-1, \operatorname{sgn}(a)-b_{2}, \ldots,-b_{s}\right)
$$

if $\left|b_{1}\right|=1$, respectively

$$
C\left(a, b_{1}, \ldots, b_{s}\right)=C\left(a-1,-\operatorname{sgn}\left(b_{1}\right), \operatorname{sgn}\left(b_{1}\right)-b_{1}, \ldots,-b_{s}\right)
$$

for $\left|b_{1}\right|>1$, such that the corresponding diagram on the right hand-side is alternating (possibly with nugatory crossings if $a-1=0$ ) and the tangle made up of the last $s$ groups therein getting the 0 -tangle by one crossing change. Hence the knot diagram unknots by that same crossing change, and we are also done in this second case.

COROLLARY 3.10. The number of almost alternating unknot diagrams of at most $n$ crossings grows exponentially in $n$.

This is some evidence against the existence of an easy classification of all such diagrams. Note, that contrarily the number of $k$-almost positive unknot diagrams grows polynomially in the crossing number for any $k$ (see [42]).

Proof. The 'at least' part of the statement follows from theorem 3.7, the uniqueness of the Conway representation with all entries even (just take $a$ and all $a_{i}$ above to be even), and the exponential growth of the number of compositions into even parts. The 'at most' part follows from the exponential growth of the total number of knot diagrams of $n$ crossings, as shown by Welsh [47].

Our final remarks concern an exact description of the counterexamples to the Bleiler conjecture for unknotting number one rational knots. In [9] he conjectured that any rational knot realizes its unknotting number in a rational diagram corresponding to the expression of its iterated fraction with all (Conway) coefficients even. This conjecture was disproved by Kanenobu and Murakami [22], quoting the counterexample $8_{14}$. We will show now exactly for which other rational knots of unknotting number one this phenomenon occurs.

LEMma 3.11. If $S(p, q)$ is an unknotting number one counterexample to the Bleiler conjecture, then it is equivalent to $S\left(2 m n \pm 1,2 n^{2}\right)$ for coprime odd numbers $m>n>1$.

Proof. The proof of this fact follows by carefully analyzing the proof of the main theorem in [22]. If $n=1$, then we have a twist knot, and if one of $m$ and $n$ is even, then the fraction $n / t$ occurring therein has an expression as iterated fraction with all integers even, and so $S(p, q)$ has an unknotting number one rational diagram with all Conway coefficients even. 
LEMMA 3.12. If $S(p, q)$ has unknotting number one and is not a counterexample to the Bleiler conjecture, then it is equivalent to $S\left(2 m n \pm 1,2 n^{2}\right)$ for coprime numbers $m>n \geq 1$, such that at least one of $m$ and $n$ is even.

Proof. From the uniqueness of the iterated fraction expression with all integers even it becomes clear that an unknotting number one rational diagram with all Conway coefficients even is of the form

$$
C\left(a, a_{1}, \ldots, a_{r}, \pm 2,-a_{r}, \ldots,-a_{1}\right),
$$

up to transposition of the integer sequence (where all $a_{i}$ are even). Then again the proof of the main theorem in [22] shows that $S(p, q) \simeq S\left(2 m n \pm 1,2 n^{2}\right)$ such that if $t$ is defined as there, at least one of $(n, t)$, and hence $(n, m)$ is even.

This leads to the following definition.

Definition 3.13. Call two coprime integers $p>q \geq 1$ irregular, if there exist coprime integers $m>n>1$ with $p=2 m n \pm 1$ and $2 n^{2} q^{ \pm 1} \equiv \pm 1 \bmod p$ (for some 3 independent sign choices ' \pm '), and in all such solution pairs $(m, n)$ both numbers are odd (where the sign choices are allowed to depend on $n$ and $m)$.

The reason for this technical definition is that it exactly tells when counterexamples to the Bleiler conjecture arise.

Proposition 3.14. $S(p, q)$ is an unknotting number one counterexample to the Bleiler conjecture iff $p$ and $q$ are irregular.

Proof. This is basically the combination of the two last lemmas, and the remark for the twist knots, that in a solution (in the sense of definition 3.13) with $n=1, m$ can be varied by \pm 1 , so can be made even.

We illustrate the fact by two examples.

ExAmple 3.15. If $m=5, n=3$ and $p=2 m n+1=31$, then $q=2 n^{2}=$ 18 and $(m, n)=(5,3)$ is a solution for $(p, q)=(31,18)$. The inverse of 18 in $Z_{31}^{*}$ is 19 , and representations of \pm 18 and \pm 19 as $2 n^{2}$ in $Z_{31}$ occur for $n=3,5,26,28$. In latter three cases $p=2 m n \pm 1>31$, and for $n=3$ the only representation of $p=2 m n \pm 1=6 m \pm 1=31$ is for $m=5$. Thus $(5,3)$ is the only solution, and, as seen already on the knot level, $S(31,18)=8_{14}$ is a counterexample to the Bleiler conjecture.

EXAMPLE 3.16. If again $m=5, n=3$, but $p=2 m n-1=29$, then again $q=18$ and $(5,3)$ is a solution for $(p, q)=(29,18)$. However, now $\left(m^{\prime}, n^{\prime}\right)=(7,2)$ is also one, because $p=2 m^{\prime} n^{\prime}+1$ and $2 n^{\prime 2} \cdot q \equiv-1 \bmod p$. Thus $(29,18)$ is not irregular and, as the reader may verify, $S(29,18)$ is not a counterexample to the Bleiler conjecture. 


\subsection{A signed version of Kanenobu-Murakami}

In this section we establish a refinement of the result of Kanenobu-Murakami [22] on unknotting number 1 rational knots.

We say that $K$ has positive unknotting number 1 (denoted by $u_{+}(K)=1$ ) if it unknots by switching a positive crossing to negative. Similarly $u_{-}(K)=1$ denotes the situation with opposite sign. These concepts were introduced in [12]. Trivially $u_{+}(K)=1 \Longleftrightarrow u_{-}(! K)=1, ! K$ being the mirror image of $K$, and $u(K)=1 \Longleftrightarrow u_{+}(K)=1$ or $u_{-}(K)=1$.

In [41] we considered knots unknotting by switching crossings of either sign and showed

Proposition 3.17 ([41]). Let $K$ be an unknotting number one knot which can be unknotted both by switching a positive and a negative crossing (not necessarily in the same diagram), and $\operatorname{det}(K)=D$. Then $\mathrm{Z}_{D}^{*}$ has square roots of -1 .

In the case of rational knots a stronger condition than Proposition 3.17 follows from the Culler-Shalen-Gordon-Luecke theorem about cyclic surgeries [14], [15], [16], applied in [22], as we show below.

Henceforth, let $\sigma$ be the signature, and $D_{K}$ denote the double branched cover of $S^{3}$ over a knot $K$. By $H_{1}=H_{1}\left(D_{K}\right)=H_{1}\left(D_{K}, Z\right)$ we denote its homology group over $\mathrm{Z}$ (the various abbreviated versions will be used at places where no confusion arises) and by $\lambda: H_{1} \times H_{1} \rightarrow \mathrm{Q} / Z$ the linking form on it. Then $\operatorname{det}(K)$ is the order of $H_{1}\left(D_{K}\right)$. There is a sign convention for $\lambda$ depending on the orientation of $D_{K}$. Our choice will be here the same as that in [43]. If we do not care about the orientation of $D_{K}$, then the following theorem is true up to interchange of ' $2 n^{2}$ ' and ' $-2 n^{2}$ '.

Theorem 3.18. Let $K=S(p, q)$ be a rational unknotting number 1 knot, which unknots by switching a positive crossing to negative, i.e. $u_{+}(K)=$ 1. Then $S(p, q) \doteq S\left(2 m n \pm 1,2 n^{2}\right)$ if $p \equiv 1 \bmod 4($ or $\sigma(K)=0)$ and $S(p, q) \doteq S\left(2 m n \pm 1,-2 n^{2}\right)$ if $p \equiv 3 \bmod 4(\operatorname{or} \sigma(K)=2)$ with $(m, n)=1$, $m>n$.

For the proof we will need one of the theorems from [43], which generalizes the theorem of [27] in an analogous way as theorem 3.18 generalizes the one of [22], and of which Proposition 3.17 is a consequence:

TheOREM 3.19 ([43]). Let $K$ be an unknotting number one knot which can be unknotted by switching a positive crossing to the negative, and $\operatorname{det}(K)=D$. Then there is a generator $g$ of $H_{1}\left(D_{K}\right)$ with $\lambda(g, g)=+2 / D \in \mathrm{Q} / \mathrm{Z}$ if $\sigma(K)=0$ and $\lambda(g, g)=-2 / D$ if $\sigma(K)=2$. 
Proof of THEOREM 3.18. First we consider the case that $p$ has a divisor $4 k+3$.

If $K=S(p, q)$, then $H_{1}\left(D_{K}\right)$ has a generator $g$ with $\lambda(g, g)=q / p$.

If $u_{+}(K)=1$, then in particular $u(K)=1$ and thus by [22] $K \sim$ $S\left(p, \pm 2 n^{2}\right)$ with $p=2 m n \pm 1$. Thus there is a generator $g^{\prime} \in H_{1}\left(D_{K}\right)$ with $\lambda\left(g^{\prime}, g^{\prime}\right)= \pm 2 n^{2} / p$.

On the other hand, theorem 3.19 implies that there is a generator $g^{\prime \prime}$ with $\lambda\left(g^{\prime \prime}, g^{\prime \prime}\right)=2 i^{p-1} / p$ (with $i=\sqrt{-1}$ used for abbreviation). Since $p$ has a divisor $4 k+3, Z_{p}^{*}$ has no square roots of -1 , and thus $\lambda\left(g^{\prime}, g^{\prime}\right)=2 i^{p-1} n^{2} / p$. Thus $K \doteq S\left(p, 2 i^{p-1} n^{2}\right)$, as desired.

Assume now $K \doteq S\left(p, 2 i^{p-1} n^{2}\right)$. Then by [22], $u(K)=1$ and moreover, $H_{1}\left(D_{K}\right)$ has a generator $g$ with $\lambda(g, g)=2 i^{p-1} n^{2} / p$. If now $u_{-}(K)=1$, by (the mirrored version of) theorem $3.19, H_{1}\left(D_{K}\right)$ would have a generator $g^{\prime \prime}$ with $\lambda\left(g^{\prime \prime}, g^{\prime \prime}\right)=-2 i^{p-1} / p$. But $g$ and $g^{\prime \prime}$ are multiples of each other, and $\mathrm{Z}_{p}^{*}$ has no square roots of -1 , a contradiction. Therefore, $u_{+}(K)=1$.

This shows the theorem in case $p$ has a divisor $4 k+3$. Now consider any odd $p>1$.

Let $u_{+}(K)=1$. Apply [22] and consider the notation $S(p, q)$ for $K$ with $p=2 m n \pm 1$ and $q= \pm 2 n^{2}$, rather than $q^{-1}= \pm 2 n^{2}$. Then for any $k \in \mathbf{Z}$, $S(p+2 k q, q)$ is also of this form, so $u(S(p+2 k q, q))=1$.

Choose $a_{i}$ as in

$$
a_{1}+\frac{1}{a_{2}+\frac{1}{\cdots+\frac{1}{a_{n}}}}=\frac{p}{q}
$$

all of positive sign. Then the diagram $D$ of $K$ corresponding to the notation $C\left(a_{1}, \ldots, a_{n}\right)$ is alternating, and by theorem 3.7 there is a crossing $c$ in $D$ whose switch unknots $K$ (we call this crossing unknotting crossing).

We claim that switching $c$ renders the rational (sub)tangle $\left(a_{2}, \ldots, a_{n}\right)$ in $D$ trivial (i.e. into the 0 -tangle). Assume this were not the case. Add $2 k$ twists to the group of crossings $a_{1}$ for $k>0$, obtaining an alternating diagram with notation $C\left(a_{1}+2 k, a_{2}, \ldots, a_{n}\right)$ corresponding to $S(p+2 k q, q)$. Since $u(S(p+2 k q, q))=1$, there must be for every $k$ a crossing $c_{k}$ in $C\left(a_{1}+\right.$ $\left.2 k, a_{2}, \ldots, a_{n}\right)$ unknotting the diagram. This crossing can not be in the group of $a_{1}+2 k$ crossings, since resolving a clasp after $c_{k}$ 's switch, we would obtain a reduced alternating diagram. Thus all $c_{k}$ belong to the tangle $\left(a_{2}, \ldots, a_{n}\right)$ for almost all $k$. But if no crossing switch makes $\left(a_{2}, \ldots, a_{n}\right)$ into the zero tangle, all these crossing switched versions of $\left(a_{2}, \ldots, a_{n}\right)$ will compose with $a_{1}+2 k$ for $k$ high enough to non-trivial rational knots, a contradiction. 
Thus there is a crossing $c$ unknotting the tangle $\left(a_{2}, \ldots, a_{n}\right)$. Since adding/removing two crossings to/from the group of $a_{1}+2 k$ crossings (or switching the signs of the crossings in this group when $a_{1}+2 k$ becomes negative under the decrease of $k$ ) does not alter the orientation of the crossings in $\left(a_{2}, \ldots, a_{n}\right)$ in the diagram $C\left(a_{1}+2 k, a_{2}, \ldots, a_{n}\right), S(p+2 k q, q)$ have either all $u_{+}=1$ or all $u_{-}=1$ for $k \in Z$.

By a classical result of Dirichlet (see e.g. [49, Korollar, p. 46]) there are infinitely many primes $s=4 k+3$, so choose one not dividing $q$. Thus there is a $k_{0}$ with $s \mid \tilde{p}:=2 k_{0} q+p$. Without loss of generality choose $k_{0}$ to be of the same sign as $q= \pm 2 n^{2}$, so that $\tilde{p} \geq p$. If now $u_{+}(S(p, q))=1$, then also $u_{+}(S(\tilde{p}, q))=1$, and thus by the already proved case $4 k+3 \mid \tilde{p}$ we have $S(\tilde{p}, q) \doteq S\left(2 m^{\prime} n^{\prime} \pm 1,2 i^{\tilde{p}-1} n^{\prime 2}\right)$. On the other hand $(\tilde{p}, q)=$ $\left(2 m n \pm 1+2\left|k_{0}\right| n^{2}, \pm 2 n^{2}\right)$. Since $Z_{\tilde{p}}^{*}$ has no square roots of -1 , we must have $(\tilde{p}, q)=\left(2 m n \pm 1+2\left|k_{0}\right| n^{2}, 2 i^{\tilde{p}-1} n^{2}\right)$. Since $2 \mid q= \pm 2 n^{2}$, we have $4 \mid \tilde{p}-p$, and thus $(p, q)=\left(2 m n \pm 1,2 i^{p-1} n^{2}\right)$, as desired.

The converse conclusion for general $p$ goes in the same way: add $2 k_{0}$ twists to $a_{1}$, use that the sign of the unknotting crossing in the alternating diagram $C\left(a_{1}+2 k_{0}, a_{2}, \ldots, a_{n}\right)$ of $S(\tilde{p}, q)$ must be positive, and undo the $2 k_{0}$ twists (not spoiling the sign of the unknotting crossing) to get back $S(p, q)$.

Remark 3.20. The proof shows also that for rational $K, u_{+}(K)=1$ if and only if its alternating diagram has a positive unknotting crossing (i.e. a positive crossing, whose switch unknots).

An application of this theorem is

PROPOSITION 3.21. The only twist knot which unknots by switching crossings of either signs (not necessarily in the same diagram) is the figure-8-knot.

Proof. Let $K$ unknot in both ways, i.e., $K$ and $! K$ both unknot by switching a positive crossing to the negative. Then $\sigma(K)=0$. By [22], $K=S(p, q)$ unknots by 1 crossing change iff

$$
S(p, q) \sim S\left(2 m n \pm_{1} 1, \pm_{2} 2 n^{2}\right)
$$

for $(m, n)=1$. If we do not care about obversion, as in [22], then both $\pm_{1,2}$ are independently choosable. However, if we fix the sign of the crossing we switch to unknot to be positive, then because of Theorem 3.18 and $\sigma(K)=0$ this means to fix the choice of $\pm_{2}$ to be ' + '. Fix also $\varepsilon \in\{1,-1\}$. Thus

$$
S(p, q) \doteq S\left(2 m n+\varepsilon,+2 n^{2}\right) .
$$

Now assume a twist knot $S(p, 2)$ with determinant $p$ and its obverse $S(p,-2)$ unknot by switching a positive crossing to the negative. Then $\sigma(S(p, 2))=0$ 
and $p \equiv 1 \bmod 4 . S(p, \pm 2)$ has the alternative representation $S(p,(p \pm 1) / 2)$ but only the negative sign is relevant for (7), as the second component of the pair is always even.

$S(p, 2)$ is trivially of the form (7), set $n=1$ and $m=(p-\varepsilon) / 2$. However, either $S(p,-2)$ or $S(p,(p-1) / 2)$ is also of the form (7). This suffices to show $p=5$, as we describe now.

First consider the case where $S(p,-2)$ is of the form (7). Then

$$
\exists k, l: \quad(k, l)=1, \quad k l=\frac{p \pm 1}{2}, \quad k^{2} \equiv-1 \bmod p .
$$

First note from the last condition that $k=1$ would lead to $p=2$, which is impossible as $p \geq 5$. If $k=2$, then $p=5$, so assume $k>2$. Also $2 l \leq p+1$ and $2 k \leq p+1$ from the middle condition in (8).

Now, because of $2 k l \equiv \pm 1(p)$ and $k^{2} \equiv-1(p)$ we have $p \mid(2 l \pm k) k$ and as $(p, k)=1$ we have $p \mid(2 l \pm k)$.

If $p \mid 2 l+k$, then $2 l \leq p+1,2 k \leq p+1$ and $k>2$ imply $2 l+k=p$. As $2 l \cdot k=p \pm 1$, we have $p \pm 1=k(p-k)$. Thus

$$
p=\frac{k^{2} \pm 1}{k-1} \leq k+1+\frac{2}{k-1} .
$$

Now $k \geq 3$ leads to $p \leq k+2$, which together with the obvious $k \leq p$ and $p \equiv \pm 1(k)$ leads to $k=3$ and again $p=5$, or to $p=k+1$ and a contradiction to the middle condition in (8).

If $p \mid 2 l-k$, then $2 l=k$ because of $2 l, k \in[2, p+1]$. But then $(l, k)=$ $k / 2=1$ leads to $k=2$, and again $p=5$.

In case $S(p,(p-1) / 2)$ is of the form (7), we need to replace the last condition in (8) by

$$
k^{2} \equiv(p-1) / 4 \bmod p .
$$

Then $p \mid 2 k(2 k \pm l)$, so $p \mid(2 k \pm l)$. If $l=1$, then we have from the middle condition in (8) that $k=(p \pm 1) / 2$, so $4 k^{2} \equiv 1(p)$, and thus with (9) that $1 \equiv$ $-1(p)$ and $p=2$, a contradiction. If $l=2$, then $k=(p \pm 1) / 4=(p-1) / 4$, but we just argued that $p$ divides $2 k \pm 2=(p-1) / 2 \pm 2$. Thus $p \leq(p+3) / 2$, a contradiction, or $(p-1) / 2-2=0$, and $p=5$. Otherwise $l>2$, and then the same argument as above applies with the role of $k$ and $l$ reversed.

\section{The HOMFLY polynomial and 2-trivadjacency}

After considering unknotting number one, we should look closer on 2-trivadjacent knots. We now consider the HOMFLY polynomial $P$. We need the following result (where $\delta$ denotes Kronecker's delta). 
Theorem 4.1 (Kneissler [26]). $X \in l^{n-1} \mathrm{Z}\left[l^{2}, l^{-2}\right]$ occurs as the lowest $m$-coefficient $[P]_{m^{1-n}}$ in the HOMFLY polynomial of an $n$ component link iff

$$
\left(l+l^{-1}\right)^{n+\delta_{n, 1}} \mid X-\left(l+l^{-1}\right)^{n-1} .
$$

We now prove

TheOREm 4.2. Let D be a diagram 2-adjacent to the unknot with crossings $c_{1,2}$.

(1) If $c_{1,2}$ are linked (i.e., the arcs in the Suzuki graph cross-hooked), then

$$
\begin{aligned}
P(D) \equiv P(C(2,1)), \quad P(C(-2,-1)) & \text { or } P(C(2,-2)) \\
& \left(\bmod m^{4}, m^{2}\left(l+l^{-1}\right)^{2}\right) .
\end{aligned}
$$

In particular, $v_{2}(D) \in\{-1,1\}$. Moreover, in the figure-8-knot case $v_{2}=-1$, we have additionally that $u_{+}=u_{-}=1$. In the positive (resp. negative) trefoil case, we have $u_{+}=1$ (resp. $\left.u_{-}=1\right)$.

(2) If $c_{1,2}$ are unlinked, then

$$
[P(D)]_{m^{0}} \equiv 1 \quad\left(\bmod \left(l+l^{-1}\right)^{3}\right),
$$

or equivalently $v_{2}(D)=0$.

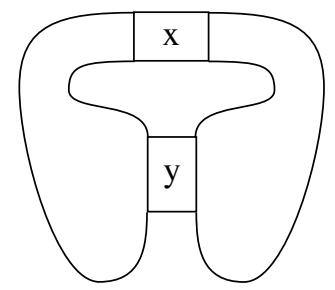

FigURE 2. A schematic picture of the $C(x, y)$ rational knot diagram. The twist knot diagrams of 3 and 4 crossings for the trefoil are those with $(x, y)=(1,2)$ and $(x, y)=(2,2)$ and the 4 and 5 crossing diagrams of the figure eight knot are those with $(x, y)=(-2,2)$ and $(x, y)=(-2,3)$, where $\pm n$ denotes $n$ positive (resp. negative) half-twists.

Here $v_{2}=1 / 2 \Delta^{\prime \prime}(1)$ is the Vassiliev invariant of degree 2 , and $C(p, q)$ is the rational knot diagram of figure 2. The coefficient of $m^{1-n}$ in the HOMFLY polynomial for an $n$ component link is sometimes called the Akutsu-DeguchiWadati polynomial (because related to their work in [2]) and will be denoted by $\Upsilon$ here. (See [29], [30] and loc. cit. for further details.) 
Proof. (1) If $c_{1,2}$ are both positive, then we have via the HOMFLY skein relation

$$
l^{-2}(P(D)-1)+\left(l+l^{-1}\right)^{2}=m^{2} P\left(D_{c_{1}(0), c_{2}(0)}\right),
$$

where $D_{c_{1}(0), c_{2}(0)}$ is the diagram obtained from $D$ by smoothing out $c_{1,2}$. If $c_{1,2}$ are linked, then $D_{c_{1}(0), c_{2}(0)}$ is a knot (and not a 3-component link) diagram, and using theorem 4.1 , we arrive to the case $P(C(2,1))$. The other 2 cases follow, when $c_{1}$ and $c_{2}$ are both negative (in which case (10) remains valid up to the change of ' $l^{-2}$ ' to ' $l$ ') ) or have opposite sign (in which case ' $l^{-2}$ ' in (10) disappears).

(2) If $c_{1,2}$ are unlinked, then $D_{c_{1}(0), c_{2}(0)}$ is a 3 component link diagram, and the statement follows again from (10) (with ' $l$-2, again possibly replaced by ' $l^{2}$ ' or omitted) and theorem 4.1.

This theorem answers a question of X.-S. Lin (see remark to question 2 in [31]) about further conditions to 2-trivadjacent knots, beside $u=1$ and $\left|v_{2}\right| \leq 1$. It can in particular show for many doubled knots with $v_{2}= \pm 1$ not to be 2-trivadjacent, like the two figure-8-knot doubles.

The signed unknotting number information in useful in the first case. Theorem 3.19 can be combined with theorem 4.2 to exclude certain knots from being 2-trivadjacent, even if they satisfy the condition on $\Upsilon=[P]_{m^{0}}$ and have unknotting number 1 . For example, $9_{34}, 10_{32}$ and $10_{147}$ have the $\Upsilon$ polynomial of the figure-8-knot. However, for $10_{147}$ we have (in Thistlethwaite's obversion convention) $\sigma=2$, so that $u_{-} \neq 1$. For $9_{34}$ and $10_{32}$ we have $\sigma=0$, but their determinant is 69 . That they cannot unknot by switching a positive and a negative crossing follows now from Proposition 3.17.

From the knots in Rolfsen's tables [38], presently the following ones have been found to be 2-adjacent to the unknot: $3_{1}, 4_{1}, 8_{17}, 8_{21}, 9_{44}, 10_{88}, 10_{136}$ and $10_{156}$. The following 19 knots are undecided: $8_{13}, 8_{14}, 9_{26}, 9_{27}, 9_{33}, 10_{30}, 10_{33}$, $10_{42}, 10_{44}, 10_{82}, 10_{87}, 10_{93}, 10_{107}, 10_{113}, 10_{114}, 10_{118}, 10_{119}, 10_{131}, 10_{146}$. The other knots are proved to be not 2-adjacent to the unknot by theorem 4.2 and its signed unknotting number consequences discussed above.

Note, that in two of the undecided 19 cases - for $10_{87}$ and $10_{93}$ - the unknotting number is not known to be one (see the tables in [24] ${ }^{1}$ ). It is unclear in how far the problem to determine 2-trivadjacency is easier or harder than to decide about unknotting number one.

Theorem 4.2 can clearly be generalized to 3 -trivadjacency (and beyond), but in this case the results of [5] (together with Suzuki's work used therein) give much more detailed statements. As mentioned in the introduction, the simplest

\footnotetext{
${ }^{1}$ The unknotting number of $10_{131}$ is one, although not indicated so there; see [43].
} 
potential examples of 3-trivadjacent knots have 14 crossings (there are two such knots, $14_{41209}$ and $14_{43932}$ in the tables of [19]). On the other hand, the apparently simplest known examples (those coming from the simplest relevant Suzuki graphs) have crossing numbers which can be estimated to be between 21 and 27, the lower bound coming from calculating the Kauffman polynomial and the (likely much closer to the actual value) upper bound obtained by applying Thistlethwaite's moves to reduce the 3-trivadjacent diagram.

\section{Everywhere 1-trivial knot diagrams (joint with N. Askitas)}

\subsection{A conjecture}

The generalization of $n$-triviality we consider now is

Definition 5.1. A knot diagram $D$ is called everywhere $n$-trivial, if for any $S \subset C(D)$ with $|S|=n, D_{S}$ is an unknot diagram.

For example, if $S=C(D)$ then $D_{S}=! D$ is the mirror image of $D$, so that in particular an everywhere $n$-trivial diagram $D$ with $|C(D)|=n$ is an unknot diagram. Another straightforward observation is that if a knot $K$ has an everywhere $n$-trivial diagram, then $u(K) \leq n$.

Here we start the investigation of everywhere $n$-trivial diagrams in the simplest case $n=1$. In [43] we made the following conjecture:

CONJECTURE 5.2. The only knots possessing an everywhere 1-trivial diagram are the unknot, trefoil and figure eight knot.

Beside the statement on knots occurring in such diagrams, it appeared appropriate to let a computer experiment clarify what everywhere 1-trivial diagrams actually occur. We describe some of the examples thus obtained, and then prove a general necessary condition such examples have to satisfy.

\subsection{Examples of everywhere 1-trivial diagrams}

5.2.1. Trefoil and figure-eight-knot. In [19], Thistlethwaite compiled tables of low crossing number knots and some programs to process these tables, which, when appropriately applied and combined, give an extremely efficient tool for examining almost any kind of question related to knot diagrams and/or knot invariants combinatorially derived from them. A collection of examples obtained this way may be found in [44].

Given the diagrams in the alternating knot tables, we have for each flype equivalence class of alternating diagrams one representative. Factoring out flypes is very appropriate when considering crossing changes, because both 
operations commute. Generating from each alternating diagram in the alternating knot tables all ${ }^{2}$ versions obtained by crossing changes and using the Jones polynomial as an (excellent) knottedness test, we obtained a list of everywhere 1-trivial diagrams of low crossing number.

In diagrams of $\leq 14$ crossings, it turns out that the trefoil occurs in the following 2 diagrams
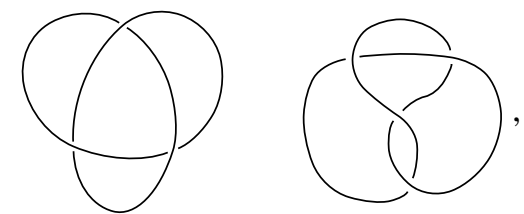

and the figure-8-knot in the following four
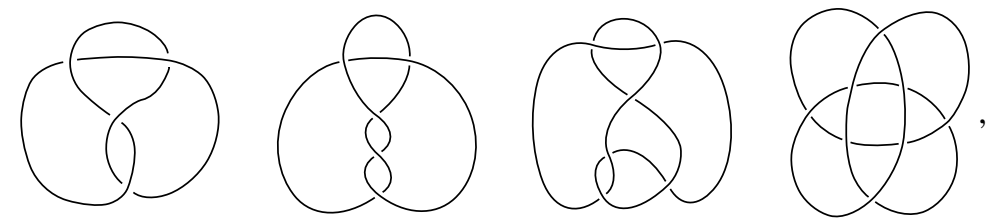

and that these are indeed the only non-trivial knots with such diagrams.

QuESTION 5.3. Do the trefoil and figure-8-knot have only the everywhere 1-trivial diagrams given in (11) and (12)?

We will later answer question 5.3 affirmatively for the trefoil (diagrams).

5.2.2. The unknot. The unknot, being the simplest knot, is of central importance to study for knot theory (see e.g. [7], [10], [32], [33]). The problem to describe, even conjecturally, everywhere 1-trivial diagrams of the unknot appears much more difficult than in the knotted cases. There is a plenty of such diagrams and their number sensibly increases with growing crossing number.

EXAMPLE 5.4. Here are four examples of everywhere 1-trivial unknot diagrams, three of them without fragments like ( (resolved clasps):
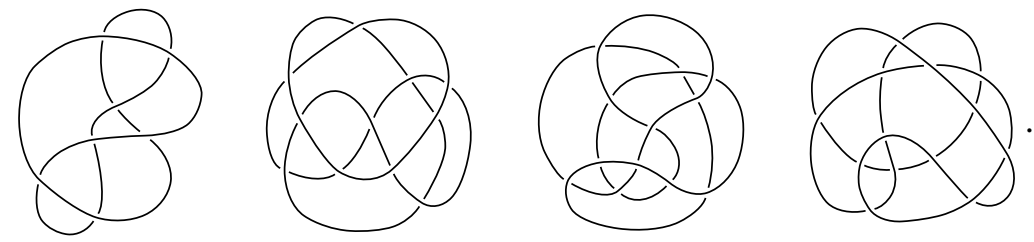

\footnotetext{
${ }^{2}$ There are many ways to reduce the number of diagrams to consider, but it would distract us too much to discuss this in detail here.
} 
We have a set of local moves to make everywhere 1-trivial diagrams more complicated. They include connected sums, if both diagrams are (everywhere 1-trivial) unknot diagrams, and the addition of trivial clasps beside a given one:

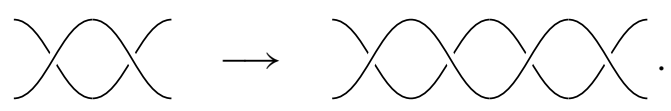

Since everywhere 1-trivial prime unknot diagrams of 6 and 7 crossings exist, the second move implies:

Corollary 5.5. Prime everywhere 1-trivial unknot diagrams of any crossing number $\geq 6$ exist.

Question 5.6. Are there prime everywhere 1-trivial unknot diagrams without a resolved clasp of every crossing number $\geq 11$ ?

ExAmPLE 5.7. To the other extreme, there are examples of unknot diagrams without a single nugatory crossing:
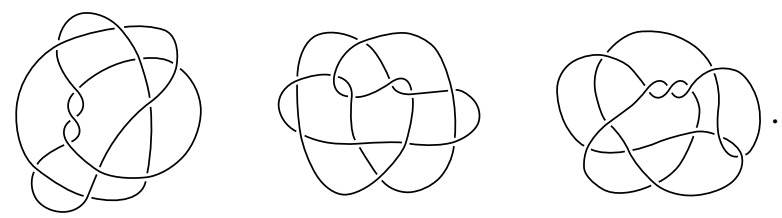

One observes that these diagrams can be turned into simpler ones by wave moves (or bridge reroutings; see [37]). This is also true for all other 12 crossing diagrams. However, there is one 13 crossing diagram which does not allow wave moves (there are several such examples with 14 crossings):

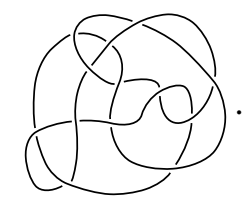

Remark 5.8. In problem 3 of X.-S. Lin's problem list [31], Zhenghan Wang asks whether two unknot diagrams with the same underlying curve can always be transformed into each other through unknot diagrams by switching single crossings each time. Examples of the above type in particular answer negatively this question. 


\subsection{A partial statement on conjecture 5.2}

We now prove a partial result towards conjecture 5.2, in particular answering positively question 5.3 for the trefoil.

THEOREM 5.9. Let $D$ be an everywhere 1-trivial diagram. Then either $D$ is the 3 or 4 crossing trefoil diagram (11), or D has the following 3 properties.

(1) $\sigma(D)=0$.

(2) $\operatorname{det}(D)$ is the sum of the squares of two coprime numbers.

(3) If $[D]$ is a twist knot, then $[D]$ is the figure-8-knot.

Proof. The first and second property would follow from $u_{+}=u_{-}=1$. If this is not the case, then $D$ is positive (or negative), and then it follows from [42] that $D$ must have (canonical Seifert surface of) genus one. From the description of such diagrams given in [42] by direct inspection we see that $D$ must be a twist knot diagram, and because of everywhere 1-triviality then it must be the 3 or 4 crossing trefoil diagram. The third property follows from proposition 3.21 .

ACKNOWLEDGEMENTS AND CREDITS. The motivation for the investigations in this paper came out of a long discussion with N. Askitas at MPI Bonn in 1999. He introduced to me the concept of $n$-trivadjacency and pointed me to the problems considered in $\S 4$. The material in $\S 5$ is obtained jointly with him following a problem of [43]; $\$ 3$ has been added subsequently and independently. (A version of the paper without $\$ 3.1$ and with more expanded $\S 5$, with N. Askitas as coauthor, was prepared, and previously circulated; $\$ 3.1$ was added from another paper [45], where it was removed in the version considered for publication following a referee's advice.)

The author would also like to thank to K. Murasugi for his careful reading of the manuscript and for pointing out some minor mistakes and simplifications in the proof of proposition 3.21 and theorem 3.18 .

\section{REFERENCES}

1. Adams, C. C., The Knot Book, W. H. Freeman \& Co., New York, 1994.

2. Akutsu, Y., Deguchi, T., and Wadati, M., Link polynomials constructed from solvable models in statistical mechanics, J. Phys. Soc. Japan 57(9) (1988), 2921-2935.

3. Askitas, N., Multi-\# unknotting operations: a new family of local moves on a knot diagram and related invariants of knots, J. Knot Theory Ramifications 7(7) (1998), 857-871.

4. Askitas, N., $\lambda$-unknotting-number-one knots need not be prime, J. Knot Theory Ramifications 8(7) (1999), 831-834.

5. Askitas, N., and Kalfagianni, E., On knot adjacency, Topology Appl. 126(1-2) (2002), 63-81.

6. Bar-Natan, D., On the Vassiliev knot invariants, Topology 34 (1995), 423-472. 
7. Bar-Natan, D., Garoufalidis, S., Rozansky, L., and Thurston, D. P., Wheels, wheeling, and the Kontsevich integral of the unknot, Israel J. Math. 119 (2000), 217-237.

8. Bernhard, J. A., Unknotting numbers and their minimal knot diagrams, J. Knot Theory Ramifications 3(1) (1994), 1-5.

9. Bleiler, S. A., A note on unknotting number, Math. Proc. Cambridge Philos. Soc. 96 (1984), 469-471.

10. Birman, J. S., and Menasco, W. W., Studying knots via braids V: The unlink, Trans. Amer. Math. Soc. 329 (1992), 585-606.

11. Burde, G., and Zieschang, H., Knots, de Gruyter, Berlin, 1986.

12. Cochran, T. D., and Lickorish, W. B. R., Unknotting information from 4-manifolds, Trans. Amer. Math. Soc. 297(1) (1986), 125-142.

13. Conway, J. H., On enumeration of knots and links, in "Computational Problems in abstract algebra" (J. Leech, ed.), 329-358. Pergamon Press, 1969.

14. Culler, M., Gordon, C. McA., Luecke, J., and Shalen, P. B., Dehn surgery on knots, Bull. Amer. Math. Soc. (N.S.) 13 (1985), 43-45.

15. Culler, M., Gordon, C. McA., Luecke, J., and Shalen, P. B., Dehn surgery on knots, Ann. of Math. 125(2) (1987), 237-300.

16. Culler, M., Gordon, C. McA., Luecke, J., and Shalen, P. B., Correction to: "Dehn surgery on knots”, Ann. of Math. 127(3) (1988), 663.

17. Gusarov, M. N., On n-equivalence of knots and invariants of finite degree, Adv. Soviet Math. 18 (1994), 173-192.

18. Freyd, P., Hoste, J., Lickorish, W. B. R., Millett, K., Ocneanu, A., and Yetter, D., A new polynomial invariant of knots and links, Bull. Amer. Math. Soc. 12 (1985), 239-246.

19. Hoste, J., and Thistlethwaite, M., KnotScape, a knot polynomial calculation program, available at http://www.math.utk.edu/ morwen.

20. Howards, Hugh, and Luecke, John, Strongly n-trivial Knots, math.GT/0004183 to appear in Bull. London Math. Soc.

21. Jablan, S. V., Unknotting number and $\infty$-unknotting number of a knot, Filomat 12(1) (1998), 113-120.

22. Kanenobu, T., and Murakami, H., 2-bridge knots of unknotting number one, Proc. Amer. Math. Soc. 96(3) (1986), 499-502.

23. Kauffman, L. H., New invariants in the theory of knots, Amer. Math. Monthly 3 (1988), 195-242.

24. Kawauchi, A., A Survey of Knot Theory, Birkhäuser, Basel-Boston-Berlin, 1996.

25. Kirby, R. (ed.), Problems of low-dimensional topology, book available on the web site http://math.berkeley.edu/ kirby.

26. Kneissler, Jan A., Woven braids and their closures, J. Knot Theory Ramifications 8(2) (1999), 201-214.

27. Lickorish, W. B. R., The unknotting number of a classical knot, Contemp. Math. 44 (1985), 117-119.

28. Lickorish, W. B. R., and Millett, K. C., A polynomial invariant for oriented links, Topology 26(1) (1987), 107-141.

29. Lieberum, J., The number of independent Vassiliev invariants in the Homfly and Kauffman polynomials, Doc. Math. 5 (2000), 275-299.

30. Lieberum, J., Chromatic weight systems and the corresponding knot invariants, Math. Ann. 317(3) (2000), 459-482.

31. Lin, X.-S., Problems in Knot Theory, http://math.ucr.edu/ xl/knotprob/knotprob.html.

32. Livingston, C., Surfaces bounding the unlink, Michigan Math. J. 29(3) (1982), 289-298.

33. Menasco, W. W., and Thistlethwaite, M. B., A geometric proof that alternating knots are nontrivial, Math. Proc. Cambridge Philos. Soc. 109(3) (1991), 425-431. 
34. Murasugi, K., Jones polynomial and classical conjectures in knot theory, Topology 26 (1987), 187-194.

35. Murakami, H., and Nakanishi, Y., On a certain move generating link-homology, Math. Ann. 284(1) (1989), 75-89.

36. Nakanishi, Y., Unknotting numbers and knot diagrams with the minimum crossings, Math. Seminar Notes Kobe Univ. 11(2) (1983), 257-258.

37. Negami, Seiya, and Okita, Kazuo, The splittability and triviality of 3-bridge links, Trans. Amer. Math. Soc. 289(1) (1985), 253-280.

38. Rolfsen, D., Knots and Links, Publish or Parish, 1976.

39. Scharlemann, M., Unknotting number one knots are prime, Invent. Math. 82 (1985), $37-55$.

40. Schubert, H., Knoten mit zwei Brücken, Math. Z. 65 (1956), 133-170.

41. Stoimenow, A., Square numbers, spanning trees and invariants of achiral knots, preprint math.GT/0003172.

42. Stoimenow, A., Knots of genus one, Proc. Amer. Math. Soc. 129(7) (2001), 2141-2156.

43. Stoimenow, A., Polynomial values, the linking form and the unknotting number, preprint.

44. Stoimenow, A., Some examples related to 4-genera, unknotting numbers, and knot polynomials, J. London Math. Soc. 63(2) (2001), 487-500.

45. Stoimenow, A., Vassiliev invariants and rational knots of unknotting number one, math.GT/9909050, Topology 42(1) (2003), 227-241.

46. Thistlethwaite, M. B., A spanning tree expansion for the Jones polynomial, Topology 26 (1987), 297-309.

47. Welsh, D. J. A., On the number of knots and links, Sets, graphs and numbers (Budapest, 1991), 713-718, Colloq. Math. Soc. János Bolyai, 60, North-Holland, Amsterdam, 1992.

48. Wendt, H., Die Gordische Auflösung von Knoten, Math. Z. 42 (1937), 680-696.

49. Zagier, D. B., Zetafunktionen und quadratische Körper, Eine Einführung in die höhere Zahlentheorie (German), Hochschultext. Springer-Verlag, Berlin-New York, 1981.

50. Zhang, X., Unknotting number one knots are prime - a new proof, Proc. Amer. Math. Soc. 113 (1991), 611-612.

WARMITZER STR. 19

13057 BERLIN

GERMANY

E-mail: stoimeno@math.fu-berlin.de,URL: http://www.math.toronto.edu/stoimeno/ 